\title{
Bull and Bear Dynamics of the Nigeria Stock Returns Transitory via Mingled Autoregressive Random Processes
}

\author{
Rasaki Olawale Olanrewaju ${ }^{1}$, Anthony Gichuhi Waititu ${ }^{2}$, Lukman Abiodun Nafiu ${ }^{3}$ \\ ${ }^{1}$ Department of Mathematical Sciences, Pan African University Institute for Basic Sciences, Technology and Innovation, Nairobi, \\ Kenya \\ ${ }^{2}$ Department of Mathematical Sciences, Jomo Kenyatta University of Agriculture and Technology, Nairobi, Kenya \\ ${ }^{3}$ Department of Mathematics and Statistics, Kabale University, Kabale, Uganda \\ Email: Rasakiolawale@gmail.com, agwaititu@gmail.com, lanafiu@kab.ac.ug
}

How to cite this paper: Olanrewaju, R.O., Waititu, A.G. and Nafiu, L.A. (2021) Bull and Bear Dynamics of the Nigeria Stock Returns Transitory via Mingled Autoregressive Random Processes. Open Journal of Statistics, 11, 870-885. https://doi.org/10.4236/ojs.2021.115051

Received: August 13, 2021

Accepted: October 16, 2021

Published: October 19, 2021

Copyright $\odot 2021$ by author(s) and Scientific Research Publishing Inc. This work is licensed under the Creative Commons Attribution International License (CC BY 4.0).

http://creativecommons.org/licenses/by/4.0/

(c) (i) Open Access

\begin{abstract}
This paper expounds the nitty-gritty of stock returns transitory, periodical behavior of its markets' demands and cyclical-like tenure-changing of number of the stocks sold. Mingling of autoregressive random processes via Poisson and Extreme-Value-Distributions (Fréchet, Gumbel, and Weibull) error terms were designed, generalized and imitated to capture stylized traits of $\mathrm{k}$-serial tenures (ability to handle cycles), Markov transitional mixing weights, switching of mingling autoregressive processes and full range shape changing predictive distributions (multimodalities) that are usually caused by large fluctuations (outliers) and long-memory in stock returns. The Poisson and Extreme-Value-Distributions Mingled Autoregressive (PMA and EVDs) models were applied to a monthly number of stocks sold in Nigeria from 1960 to 2020. It was deduced that fitted Gumbel-MAR $(2: 1,1)$ outstripped other linear models as well as best fitted among the Poisson and Extreme-ValueDistributions Mingled autoregressive models subjected to the discrete monthly stocks sold series.
\end{abstract}

\section{Keywords}

Autoregressive Random Processes, Extreme-Value-Distributions, Mingled, Poisson, Stock Returns

\section{Introduction}

Volume traded and volatility are two key concepts in finance and marketing/trading of stock. The association between the stock volume traded and its fluctuation do provide deep introspection into organizing financial stock mar- 
kets, switching of the price, size of the market, number of prospective or continuous buyer and information flow [1].

Market tales, legends and superstitions hold the view that volume-price relationship movements conditioned on whether the market is in a bull (situation that market demand endeavors to raise price market) or bears (of a market in which prices are rising systematically) control process. As bull market is concerned, a comparatively eminent volume will be uniting with an agreed price alteration compared to bear market settings. It is with this reason some technical analysts gave a verdict that less significance should be given to raise price associated with relatively low marketing volume compared to rising in similar price as well as significant volume [2]. Moreover, trading volume in relation to its volatility has also provided deep introspection into cohesive buildup of financial markets that was found to provide insight as per magnitude or relative frequency of information fluxed in the mart place, constituted short stock market, market expansion, and level to which prices manifest needed info by the public [3].

There are several existing methods and techniques used to estimate and forecast asset prices, number of stocks sold, overpriced securities or stock to safeguard investments. Four of the traditional methods used to assess stock market prices, total number sold at a particular period and subsidize buying and selling orders by financial investors are: fundamental analysis, which consists of analyzing a coherent set of accounting numbers and financial ratios to assess the financial dispersion (volatility), regime-switching, generalization of the uniform time model that would lead to the choice of investment options in the market [4] [5].

Among the numerous models employed in predicting stock analyses was Moving Average (MA) model via an exponentiated distribution, known as EMA. However, according to [5], numerous forecast models mandated stationarity process of any observational financial stock series. In real sense, these series are usually non-stationary process; consequently, autoregressive models had been ascertained to modify the non-stationarity by adding time-dependent series. Testing market performances of stock and prototyping stock prices have been the studying of two wide broad areas of stock market changes. Efficient Market Hypothesis (EMH) has been the test statistic for testing market performances of stock with disentail of stock prices. Several generalization techniques and derivations have been employed to model Stock Market Index (STM) prices with few cognitive taken on modelling the number of stocks sold at a uniform time interval not alone of subjecting the number of stocks sold at uniform period to a regime-switching model such as Mingle Autoregressive (MAR) model. A number of stocks sold at a constant interval uniform of time are not randomly generated values, but are well-described numerical observations gathered at constant interval of time need to be treated as discrete type of time series model. Having ascertained the type discrete time series model, linear models might not be competing ones because they are statistically built for linear Gaussian models; and mostly provided first-order approximations processes [6]. However, the 
one-way volatility series could not be able to describe the financial series because of its returns exhibition of changes in transitory (jumps), outliers, clusters, usual possession of changing behavior of stock market for many periods (stock returns cyclical change), autocorrelations of stock returns across regimes, which will allow the stochastic tenure-changing model to represent stylized traits of the number of stock market series as well as heavy-tails, heteroscedasticity, and time-changing correlations [7].

However, stock market (number of stocks sold at a constant interval uniform of time) exhibited high variability, business cycle dependence, a mixture tenure-changing model will be adopted to represent the aforementioned traits in support with a discrete random noise (distributional error term) that will cater for the count series, changing means-variances (volatilities) heteroscedasticity, and time-varying correlations for the number of stocks sold monthly. This article proposed two category of mingling autoregressive random processes-Poisson and Extreme-Value-Distributions (Fréchet, Gumbel, and Weibull). The two categories of processes will be designed to capture stylized traits of k-serial tenures of monthly number of stocks sold in Nigeria from 1960 to 2020 and analyze qualitatively and quantitatively.

\section{Literature Review}

Recently, [8] illustrated the Gaussian-MAR (GMAR) model mathematically and analytically. The data used was retrieved from OECD Statistics, a monthly dataset between January, 1989 and December, 2009 for differences in Euro adopted by regions and U.S. long-term government bond production of specific amount that contained instability of the fiscal abrupt change in 2008. They presented analytically the GMAR with the stock returns series. It was claimed that the stock returns were naturally eminent reoccurrence and exhibited non-linearity behavior perhaps as a result of tenure changing dynamics.

[9] examined the relationship between Heterogeneous Autoregressive Realized Volatility (HAR-RV) model and extended it to Heterogeneous Autoregressive endogenous variable Realized Volatility (HARX-RV) model. [9] research was designed for correlational study for 20 companies forming the NSE 20-share index, the stock prices at each closing day for all the NSE 20-share companies comprising the index and daily trade volume between January, 2008 and December, 2013. He computed the daily realized volatility via standard deviation and realized volatility at different time horizons. He applied ordinary least squares regression and autoregression on the share index and daily trade volume on the study. The study used F-statistic valued at 39.4597 and 30.0461 for HAR-RV and HARX-RV models respectively, which indicated that the models were statistically significant. Results from HAR-RV model show that fluctuation of stock returns was reoccurrence in NSE and the persistence reduces when volume is added to the model.

Furthermore, [10] examined the changes of the monthly effect (seasonal patterns usually found on global financial series) on stock returns in twenty coun- 
tries (selected from different continents: Asia, Europe, Australia, America) using symmetric Autoregressive Conditional Heteroscedasticity (ARCH) and Periodic-GARCH. They adopted the Ordinary Least Squares (OLS) as the parameter estimation technique to checkmate the problem of unreliable estimations that might arise from ARCH parameterization effects. [10] deduced that symmetric $\mathrm{ARCH}(1,1)$ model was the optimal such that the estimated variation inclines to add-up more whenever the STM diminishes than whenever the STM adds-up by similar amount. Additionally, [10] found that there was eminent seasonality effect in variation instead of mean repays; due to this, Periodic-GARCH $(1,1)$ was estimated. His findings supported the doggedness of the particular calendar consequence in nineteen out of twenty countries studied.

[11] raised a critical lacuna about the statistical related bureaus and central bank in countries that publish seasonal adjustment economic information of mingle frequency stock and continuous progression time series. Their concern was the problem of computational imputations, forecasts, non-stationarity, model estimation, and backcast from a used model to available frequency data. They carefully formulated and designed computations that could allow to estimate needed coefficients via Logarithm-Normal likelihood for the mingled frequency stocks and flow time series, in addition to attribution, predictions and derived applicable median absolute error. They evaluated the methodology via simulations and mixed frequency stock techniques of some economic time series. [2] studied the effect rate of change of prices (as indicated by a price index) estimated on monthly basis on stock returns in the Nigeria stock market, and determined if rate of change of prices have impact on stock market returns, as well determined if prices of stocks affect forecast of stock returns. They used the method of empirical analysis via Autoregressive Distributed Lag (ARDL) technique to estimate the connectedness between stocks repays and rate of change of prices. They used the monthly stock repays and rate of change of prices covering the period between 1995 and 2010. The dataset was extracted from the Nigeria Stock Exchange (NSE) factual hand book in collaboration with the Central Bank of Nigeria's newssheet respectively. Their analysis indicated that the rate of change of prices has a weak, but oppositional effect stock returns; hence, rate of change of prices has a weak forecast stock returns in Nigeria.

[3] investigated the restriction of ARIMA model in fiscal and pecuniary economics using the attribute of BET stock index and Euro-ron exchange rates. They discovered two crucial traits possessed by financial series are heavy-tails and fluctuation clustering, which empirical cannot be represented or expressed by incorporated ARMA models as limitation of ARIMA models.

[12] analyzed the NSE All Share Index with dataset obtained from CBN's newsletter. The dataset used was made up of three hundred fifty-seven observations of a dataset recorded at every last Friday of working day of every Friday of working day of every month from January, 1985 to September, 2014. They adopted the Box and Jenkins steps of constructing ARIMA model via identification of model, estimating of parameters, and diagnostic checking respectively. It was 
unveiled that the differencing autoregressive model of order two AR (2) gave the optimal order for with Alkaike Information Criteria (AIC) of 6682.4416 for the NSE All Share Index, that is, $X_{t}=0.47 X_{t-1}+0.5123 X_{t-2}$. They concluded that, ARIMA $(2,1,0)$ was the requirement optimization to generalized the NSE All Share index.

[7] examined the insufficiency of fallible expression level within the Swedish stock trade by using GARCH and its variant, Threshold-GARCH (TGARCH) to inquiry if every day of the week marketing activities in Swedish index OMXS30 effect introduced in 1970s has improved market efficiency. The everyday of the week marketing activities effect was studied between the period of 2000 and 2017. A quantitative analysis was conducted on the OMXS30 via TGARCH and GARCH and results revealed that every day of the week marketing activities impact not manifested within the OMXS30 during this period, furnishing grounds for improvement in market efficiency.

Several models have been formulated over time to capture correctly the stylized facts of number of stocks sold (stock returns) in a constant uniform time interval. On the other hand, these reviewed models were unable to express and represent the features associated to stock returns, that especially exhibited high variability, business cycle dependence, mixture regime-switching associated to stock returns. In addition, these reviewed models failed to realize that series of number of stocks sold ought to be in line with strictly count random noise (error term), like that of Poisson regression model as well as that of Extreme-ValueDistributions that cater for fluctuations, tenure-switching and domain range of $(-\infty, \infty)$ values in application to Mingle Autoregressive (MAR) model. In a nut shell, this paper would be subjecting the number of stocks sold to regimeswitching MAR model with Poisson and Extreme-Value-Distributions (Fréchet, Gumbel, and Weibull) probabilistic distributions.

\section{Specification of the Mingle Autoregressive Processes}

[13] propounded that a finite (countable) mingle time series with similar probabilistic distribution as a generalization of;

$$
g(y)=\eta_{1} f_{1}(y)+\eta_{2} f_{2}(y)+\cdots+\eta_{k} f_{k}(y)
$$

Such that $g(y)$ is the complete function of the tenure-switching generalization model, with $f_{i}(y)(i=1, \cdots, k)$ identical probabilistic distribution per each tenure-switching ${ }_{k}$ with their corresponding Markov mixing transitional weights of $\eta_{i}, \eta_{i}>0$ э $\sum_{i=1}^{k} \eta_{i} \approx 1, i=1, \cdots, k$.

Otherwise,

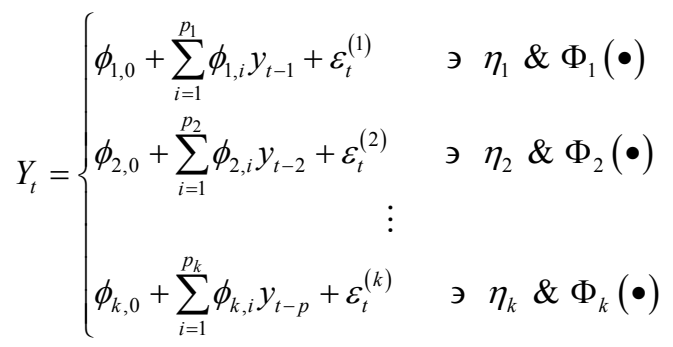


where, $\phi_{p k} \in(0,1), \quad p \geq 1$ for $k=1, \cdots, K$ which represents the mingling autoregressive coefficients per each tenure. $\Phi_{k}(\bullet)$ is/are the " $k$ " cyclical distributional parameter (s). For weighted transitional probabilities $\eta_{1}+\cdots+\eta_{k} \approx 1$, $\eta_{i}>0$, for $k=1, \cdots, K$, such that, the error terms, $\varepsilon_{t}^{(k)}$ follows:

1)

$$
\begin{gathered}
f\left(y_{t} ; a_{k}, b_{k}\right)=\frac{a_{k}}{b_{k}}\left(\frac{b_{k}}{y_{t}}\right)^{b_{k}+1} \exp \left(-\left(\frac{b_{k}}{y_{t}}\right)^{a_{k}}\right), \quad y_{t}>0 \\
f\left(y_{t} ; b_{k}, \mu_{k}\right)=\frac{1}{b_{k}} \exp (-\pi-\exp (-\pi)),-\infty<y_{t}<+\infty
\end{gathered}
$$

where, $\pi=\frac{y_{t}-\mu_{k}}{b_{k}}$

$$
f\left(y_{t} ; a_{k}, b_{k}\right)=\frac{a_{k}}{b_{k}}\left(\frac{y_{t}}{b_{k}}\right)^{a_{k}-1} \mathrm{e}^{-\left(\frac{y_{t}}{b_{k}}\right)^{a_{k}}}, y_{t} \geq 0
$$

Equations (3) to (5) stands for Fréchet, Gumbel, and Weibull Probability Density Functions (PDFs) respectively. The $y_{t}$ in each equation is the cyclical stock returns series that each represents the Fréchet Mingle Autoregressive (FMA), Gumbel Mingle Autoregressive (GMA) and Weibull Mingle Autoregressive (WMA) models. $\Phi_{k}(\bullet)$ in each FMA, GMA, and WMA model denotes their respective switching distributional parameters, $a_{k}>0$ (the shape parameter per each regime), $b_{k}>0$ (the scale parameter per each regime); $\mu_{k}$ (the location parameter per each regime). The three distributions make-up the Extreme-Value-Distributions to give Extreme-Value-Distributions'-MingleAutoregressive model.

2)

$$
f\left(y_{t} ; \lambda_{k}\right)=\frac{\lambda_{k}^{y_{t}} \mathrm{e}^{-\lambda_{k}}}{y_{t} !}, y_{t}=0,1,2,3, \cdots
$$

Equation (6) stands for the Poisson Probability Mass Function (PMF). The $y_{t}$ in Equation (5) is the cyclical stock returns series that make it represents Poisson Mingle Autoregressive (PMA) for strictly count (discrete) noisy series.

\section{Procedure for the Parameter Estimation}

Suppose $Y=\left\{Y_{1}, Y_{2}, \cdots, Y_{n}\right\}^{\mathrm{T}}$ and $M$ is an unobserved (latent) variable, that is, unmeasured variable, where $M_{t}$ is a $k$-dimensional quantity э $M_{t}=\left\{M_{1}, M_{2}, \cdots, M_{t}\right\}^{\mathrm{T}}$, whose component is a dichotomous of $M_{i, t}=1$ if $Y_{t}$ is a member of the $j^{t h}$ tenure, while $M_{i, t}=0$ if otherwise, $\ni 1 \leq j \leq k$, then,

$$
\begin{gathered}
\eta_{1}=P\left(M_{t}=(1,0, \cdots, 0)^{\mathrm{T}}\right) \\
\eta_{2}=P\left(M_{t}=(0,1, \cdots, 0)^{\mathrm{T}}\right) \\
\vdots \\
\eta_{k}=P\left(M_{t}=(0,0, \cdots, 1)^{\mathrm{T}}\right)
\end{gathered}
$$


Let $a_{k}=\left\{a_{0}, a_{1}, \cdots, a_{k}\right\}^{\mathrm{T}} ; b_{k}=\left\{b_{0}, b_{1}, \cdots, b_{k}\right\}^{\mathrm{T}} ; \phi_{k}=\left\{\phi_{k 0}, \phi_{k 1}, \cdots, \phi_{k p k}\right\}^{\mathrm{T}}$; $\eta_{k}=\left\{\eta_{0}, \eta_{1}, \cdots, \eta_{k}\right\}^{\mathrm{T}}$ for $k=1, \cdots, K$.

Suppose $M_{t}$ is identified, the observed and unobserved data for the Fréchet and Weibull distributions in Equations (3) and (5) can be written as $\left(Y_{t}, M_{t}\right)$, such that their maximizing conditional log-likelihood functions give:

$$
\begin{gathered}
L_{t}(\Omega)=\log \prod_{k=1}^{K}\left[\eta_{k} \frac{a_{k}}{b_{k}}\left(\frac{b_{k}}{Y_{t}}\right)^{a_{k}+1} \exp \left(-\left(\frac{b_{k}}{Y_{t}}\right)^{a_{k}}\right)\right]^{M_{k t}} \\
L_{t}(\Omega)=\log \prod_{k=1}^{K}\left[\eta_{k} \frac{a_{k}}{b_{k}}\left(\frac{Y_{t}}{b_{k}}\right)^{a_{k}-1} \exp \left(-\frac{Y_{t}}{b_{k}}\right)^{a_{k}}\right]^{M_{k t}}
\end{gathered}
$$

where $\Omega=\left\{\phi_{k}, \eta_{k}, a_{k}, b_{k}\right\}^{\mathrm{T}}=\left\{\phi_{k}, \eta_{k}, \Phi_{k}(\bullet)\right\}^{\mathrm{T}}$ is the parameter space, $\Phi_{k}(\bullet)$ is the distributional parameters of the Fréchet and Weibull distributions.

In a similar vein,

Let $b_{k}=\left\{b_{0}, b_{1}, \cdots, b_{k}\right\}^{\mathrm{T}} ; \mu_{k}=\left\{\mu_{0}, \mu_{1}, \cdots, \mu_{k}\right\}^{\mathrm{T}} ; \phi_{k}=\left\{\phi_{k 0}, \phi_{k 1}, \cdots, \phi_{k p k}\right\}^{\mathrm{T}}$; $\eta_{k}=\left\{\eta_{0}, \eta_{1}, \cdots, \eta_{k}\right\}^{\mathrm{T}}$ for $k=1, \cdots, K$

Suppose $M_{t}$ is identified, the observed and unobserved data for the Gumbel distribution in Equations (4) can be written as $\left(Y_{t}, M_{t}\right)$, such that its maximizing conditional log-likelihood function gives:

$$
L_{t}(\Omega)=\log \prod_{k=1}^{K}\left[\eta_{k} \frac{1}{b_{k}} \exp \left(-\frac{\left(Y_{t}-\mu_{k}\right)}{b_{k}}-\exp \left(-\frac{\left(Y_{t}-\mu_{k}\right)}{b_{k}}\right)\right)\right]^{M_{k t}}
$$

where $\Omega=\left\{\phi_{k}, \eta_{k}, b_{k}, \mu_{k}\right\}^{\mathrm{T}}=\left\{\phi_{k}, \eta_{k}, \Phi_{k}(\bullet)\right\}^{\mathrm{T}}$ is the parameter space, $\Phi_{k}(\bullet)$ is the distributional parameters of the Gumbel distribution.

Lastly, for the discrete (count) series using the Poisson PMF.

Let $\lambda_{k}=\left\{\lambda_{0}, \lambda_{1}, \cdots, \lambda_{k}\right\}^{\mathrm{T}} ; \phi_{k}=\left\{\phi_{k 0}, \phi_{k 1}, \cdots, \phi_{k p k}\right\}^{\mathrm{T}} ; \eta_{k}=\left\{\eta_{0}, \eta_{1}, \cdots, \eta_{k}\right\}^{\mathrm{T}}$ for $k=1, \cdots, K$.

Suppose $M_{t}$ is identified, the observed and unobserved data for the Poisson distribution in Equation (6) can be written as $\left(Y_{t}, M_{t}\right)$, such that its maximizing conditional log-likelihood function gives:

$$
L_{t}(\Omega)=\prod_{k=1}^{K}\left[\eta_{k} \frac{\lambda_{k}^{Y_{t}} \mathrm{e}^{-\lambda_{k}}}{Y_{t} !}\right]^{M_{k t}}
$$

where $\Omega=\left\{\phi_{k}, \eta_{k}, \lambda_{k}\right\}^{\mathrm{T}}$ is the parameter space, $\lambda_{k}$ is the distributional parameters of the Poisson distribution.

The Extreme-Value-Distributions'-Mingle-Autoregressive (EVDs-MAR) and Poisson Mingle Autoregressive models' (PMA) in Equations (8), (9) and (10) parameter estimation respectively can be carried-out via Weighted Iterative Generalized Least Square (WIGLS) or via Expectation-Maximization (EM) technique, see [14] [15] [16].

\section{Numerical Results of Application}

The parametric solutions of the Extreme-Value-Distributions'-Mingle-Auto- 
regressive and Poisson Mingle Autoregressive models' parameter estimations of the maximizing conditional log-likelihood functions in Equations (8) - (10) will be applied to monthly number of stocks sold in Nigeria from 1960 to 2020, as received from the Nigerian Stock Exchange (NSE). The discrete data will be applied to both categories of models for comparison and evaluation.

From Figure 1, it is obvious that the first ten years (1960-1970) monthly number of stocks sold swing between 250 and 320 before a prolong and sporadic rise to apex of around 920 was experienced around 1972 to 1974 . The stocks' swing maintained number of stocks from 270 to 850 between 2008. At the beginning of 2009, constant skyrocketed in the increment to around 1000 stocks sold monthly till mid-2011 was maintained, before a continuous drastic fall to around 350 monthly from 2012 to 2017 . A miniature continuous fall to 250 consecutively from 2018 to 2020 was experienced.

From Table 1, it can be inferred that there is a large dispersion in the stock dataset for the seventy years studied because the difference in the largest and smallest value of monthly number of stocks was moderately large. The mean value of 1990.5 for the stock dataset implied that the recorded monthly number of stocks sold clustered around 1990.5. It is to be noted the mean value is not among the monthly observation, suggesting a wide deviation among the dataset. The median value of 1990.5 coincides with the mean value, this implies that half of the monthly stocks sold are less than or equal to 1990.5, and half monthly stocks sold are greater than or equal to 1990.5. However, since the difference in the mean and median values is zero, it indicated that there is chance for the distribution of the data to be symmetric if the mode value coincides as well.

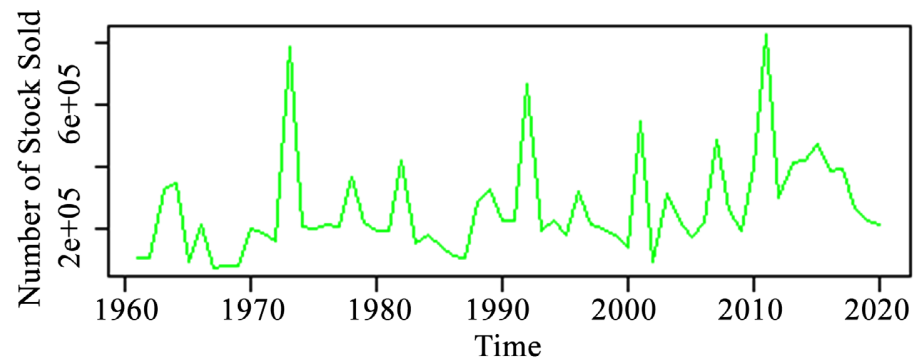

Figure 1. Time plot for monthly number of stock sold.

Table 1. Explanatory Data Analysis (EDA) for the monthly number of stocks sold.

\begin{tabular}{|c|c|c|c|c|c|c|c|c|}
\hline Statistic & \multicolumn{2}{|c|}{ Range } & Mean & Variance & $\begin{array}{c}\text { Std. } \\
\text { Deviation }\end{array}$ & $\begin{array}{l}\text { Coef. of } \\
\text { Variation }\end{array}$ & $\begin{array}{l}\text { Std. } \\
\text { Error }\end{array}$ & Max. \\
\hline Stock & \multicolumn{2}{|c|}{590} & 1990.5 & 305 & 17.464 & 0.0088 & 2.2546 & 2020 \\
\hline \multicolumn{9}{|c|}{ Percentiles } \\
\hline Percentile & Min. & $5 \%$ & $10 \%$ & $\begin{array}{l}25 \% \\
\text { (Q1) }\end{array}$ & $\begin{array}{c}50 \% \\
\text { (Median) }\end{array}$ & $\begin{array}{l}75 \% \\
\text { (Q3) }\end{array}$ & $90 \%$ & $95 \%$ \\
\hline Stock & 1961 & 1963.1 & 1966.1 & 1975.3 & 1990.5 & 2005.8 & 2014.9 & 2018.0 \\
\hline
\end{tabular}


The standard deviation of 17.464 for the stipulated years of study indicates high proximity in the individual records to be estimated. However, since the estimated Coefficient of Variation is $0.88 \%$, it connotes that there is closeness in the estimated mean and standard deviation to their true population values. For the $5^{\text {th }}, 10^{\text {th }}, 25^{\text {th }}, 75^{\text {th }}, 90^{\text {th }}$, and $95^{\text {th }}$ percentiles, it implies that $5 \%, 10 \%, 25 \%$, $75 \%, 90 \%, 95 \%$ of the monthly number of stocks sold for the studied period are $1963.1,1966.1,1975.3,2005.8,2014.9,2018.0$ or below and that of $95 \%, 90 \%$, $75 \%, 25 \%, 10 \%$ and $5 \%$ of the monthly number of stocks sold for the studied period are above 1963.1, 1966.1, 1975.3, 2005.8, 2014.9, 2018.0 respectively.

According to the rule of thumb of coefficients of skewness that says if it is $<-1$ or $>+1$, it indicated highly skewed. From Table 2, the coefficients of skewness of 1.6045 greater than +1 implies that there is strong indication that the data is skewed. Using the rule of thumb for interpreting coefficients of kurtosis, since the coefficient of kurtosis for the monthly number of stocks sold is $2.7801<3$, it indicates a platykurtic distribution with tail (s) (either unimodal, bimodal or multimodal) shorter and thinner with often lower and broader central peak. However, since the p-value for Shapiro-Wilk test for the monthly number of stocks sold is $0.0045<\alpha$-level $=0.05$, then we fail to accept that the dataset came from a normal distribution. Similarly, since a large chi-square statistic was estimated with p-value $=0.0227<0.05$, we fail to accept that the dataset is normally distributed. On the same account, since the ratio of the variances $0.05,0.03$, 0.0022 and 0.0002 for Gumbel, Fréchet, Weibull and Poisson distributions respectively are not approximately equal to one, it implies that the variance from each distributional dataset is either too high or too low, indicating either negative or positive autocorrelation via their distributional fittings. Moreover, since the ADF statistic of -3.1967 for the monthly number stocks sold is negative coupled with the fact that its p-value $=0.0965>0.05$, we accept the null hypothesis that there is a unit root at $5 \%$ level of confidence.

From Table 3, literally fitting the stock dataset to Gumbel, Fréchet, Weibull, Normal and Poisson distributions each without considering of any type of time-varying random process, it is glaring that Gumbel distribution has the true and best fit (ideal representation) with the most beneficial model performance of $\mathrm{AIC}=1586.1, \mathrm{BIC}=1590.289$ compared to $\mathrm{AIC}=1588.65, \mathrm{BIC}=1592.839 ; \mathrm{AIC}$ $=1592.709, \mathrm{BIC}=1596.898 ; \mathrm{AIC}=1610.27, \mathrm{BIC}=1614.458 ; \mathrm{AIC}=48,346, \mathrm{BIC}$ $=48,358$ for Fréchet, Weibull, Normal and Poisson distributions respectively. There seems to be a weak positive correlation between the two parameters (scale and shape) in each of the distribution, except for normal distribution with

Table 2. Normality test and EVDs-variance ratio test for the monthly number of stocks sold.

\begin{tabular}{cccccccccc}
\hline Statistic & Skewness & Kurtosis & Shapiro-Wilk & Jarque-Bera & V. R. Gumbel & V.R. Frechet & V.R. Weibull & V. R. Poisson & A.D.F \\
\hline Stock & 1.6045 & 2.7801 & 0.84245 & 49.243 & 0.7356 & 1.1897 & 0.7356 & 0.7856 & -3.1967 \\
p-value & - & - & 0.0045 & 0.0227 & 0.05 & 0.03 & 0.0022 & 0.0002 & 0.0965 \\
\hline
\end{tabular}


strong positive correlation between its shape and location parameters. The Kolmogorov Smirnov goodness-of-fit test for continuous distributions was adopted to test whether truly the number of stocks sold agents' datasets came from the EVDs, came from any of the EVDs or Poisson distribution.

In a similar vein, the $\mathrm{K}-\mathrm{S}$ test statistic $\mathrm{D}=0.1072, \mathrm{D}=0.0970, \mathrm{D}=0.08015, \mathrm{D}$ $=0.0636>0.04301$ (critical value at $\alpha=5 \%$ ) for Gumbel, Fréchet, Weibull (EVDs) and Normal distributions, it implies the number of stocks sample came from EVDs while there is no sufficient evidence to attach sample to a Poisson distribution, because $\mathrm{D}=0.004<0.04301$ (Figure 2).

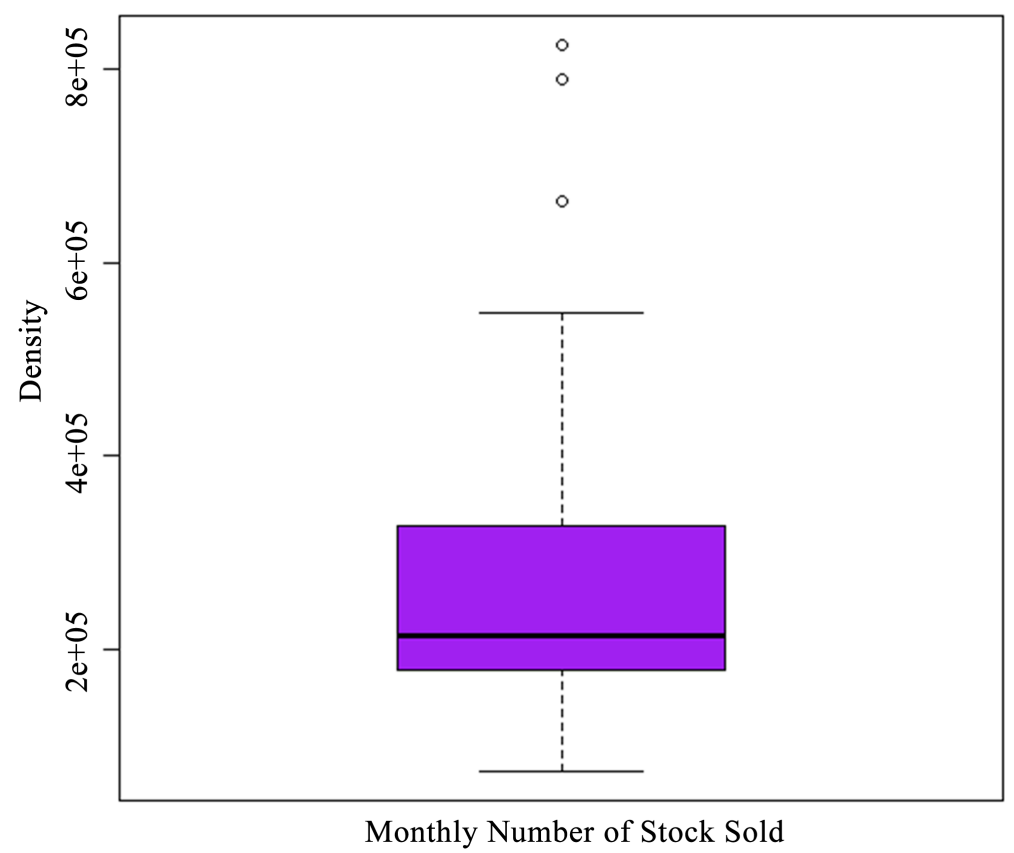

Figure 2. Boxplot for the monthly number of stocks sold.

Table 3. Estimate of the distributional parameters and goodness-of-fit test for the fitted stock of EVDs, normal and Poisson.

\begin{tabular}{|c|c|c|c|c|c|c|c|c|}
\hline & Estimates & $a / \mu$ & $b$ & $\operatorname{cor}(a / \mu, b)$ & Loglik. & AIC & BIC & K. S. Test Statistic \\
\hline \multirow{7}{*}{ Stock } & Gumbel & $\begin{array}{c}13.617 \\
(0.3521)\end{array}$ & $\begin{array}{c}1982.6 \\
(0.0674)\end{array}$ & 0.3551 & -791.05 & 1586.1 & 1590.289 & 0.1072 \\
\hline & Fréchet & $\begin{array}{c}1.9206 \\
(0.0004)\end{array}$ & $\begin{array}{l}258.815 \\
(0.0839)\end{array}$ & 0.2789 & -792.325 & 1588.65 & 1592.839 & 0.0970 \\
\hline & Weibull & $\begin{array}{c}1.8269 \\
(0.0028)\end{array}$ & $\begin{array}{l}446.548 \\
(0.0006)\end{array}$ & 0.4309 & -794.355 & 1592.709 & 1596.898 & 0.08015 \\
\hline & & $\mu$ & $\sigma$ & & & & & \\
\hline & Normal & $\begin{array}{c}1990.5 \\
(0.0427)\end{array}$ & $\begin{array}{c}17.464 \\
(0.0232)\end{array}$ & 0.5671 & -803.135 & 1610.27 & 1614.458 & 0.0636 \\
\hline & & $\lambda$ & & & & & & \\
\hline & Poisson & $\begin{array}{c}1990.5 \\
(65.536)\end{array}$ & - & - & -24173 & 48346 & 48358 & 0.0045 \\
\hline
\end{tabular}


From the boxplot (Box whisker plot) above, the median (the black line) is closer to the bottom box with the whisker slightly shorter at the lower end of the box, this suggested a possibly left-skewed (negatively skewed) distribution for the monthly number of stocks sold within the seventy years of study. Moreover, since there are points outside the whisker of the boxplot, it means there are some numerical values far distanced from others. In other words, there are three different points (referred to as outliers or anomalies) to the outside of the whisker of the boxplot.

From Figure 3, the histogram for monthly number of stocks sold dataset looks like a lopsided mound, with the tail going-off to the left. It means a left-skewed distribution with the shape of the histogram showing three modes (tri-modal).

In a similar vein to that of the histogram plot 3, Figure 4 shows higher resolution details of tri-modal, bimodal for Normal, Gumbel, and Fréchet distributions respectively, while unimodal were associated to Weibull and Poisson distributional densities.

From Table 4, the two-regime SETAR model was at its best for the monthly number of stocks sold dataset with Gumbel random noise, optimally at Gumbel-SETAR $(2: 2,1)$ with AIC $=-7938$; BIC $=-7955$; MAPE $=21.29 \%$ such that, the discrete monthly number of the series was partitioned into $16.31 \%$ for the low-regime and $83.69 \%$ for the high-regime at Gumbel-SETAR $(2: 2,1)$. Meaning, the optimal autoregressive order for low-regime and high-regime was at order 1 and 2 respectively.

Narrowing it down to MAR models, MAR with Gaussian, that is, Norm-MAR $(3: 2,1,3)$ piled-up a very high AIC and BIC of 1517.1827 and 1523.166 respectively

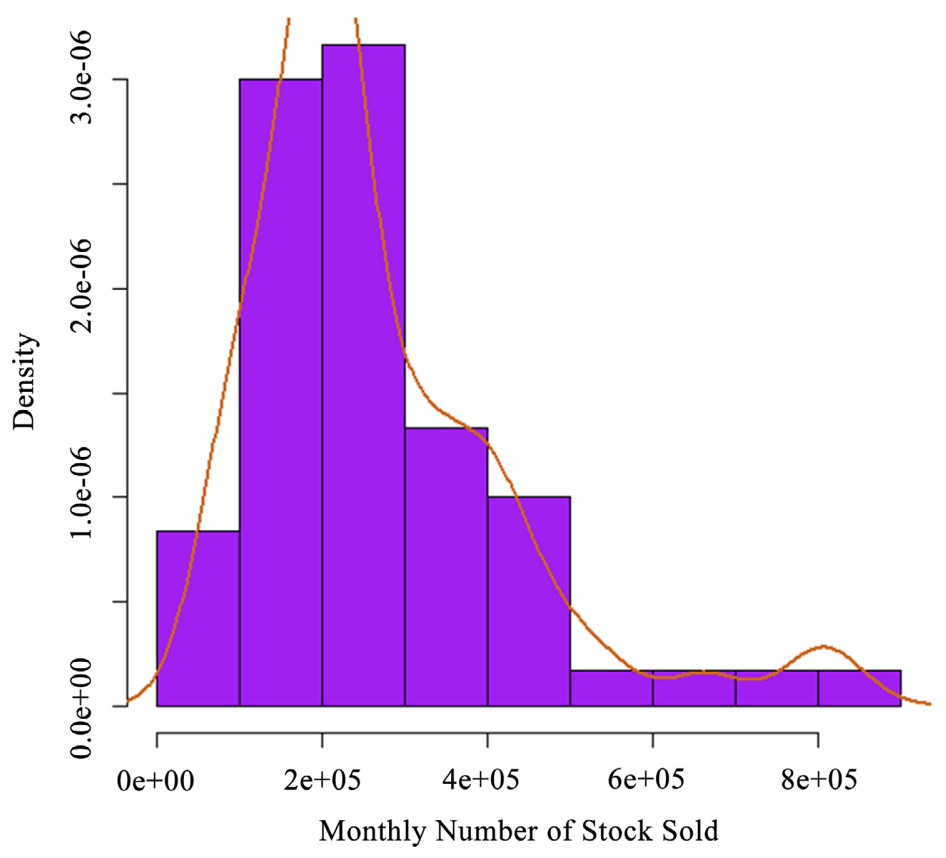

Figure 3. Histogram plot for the monthly number of stocks sold. 

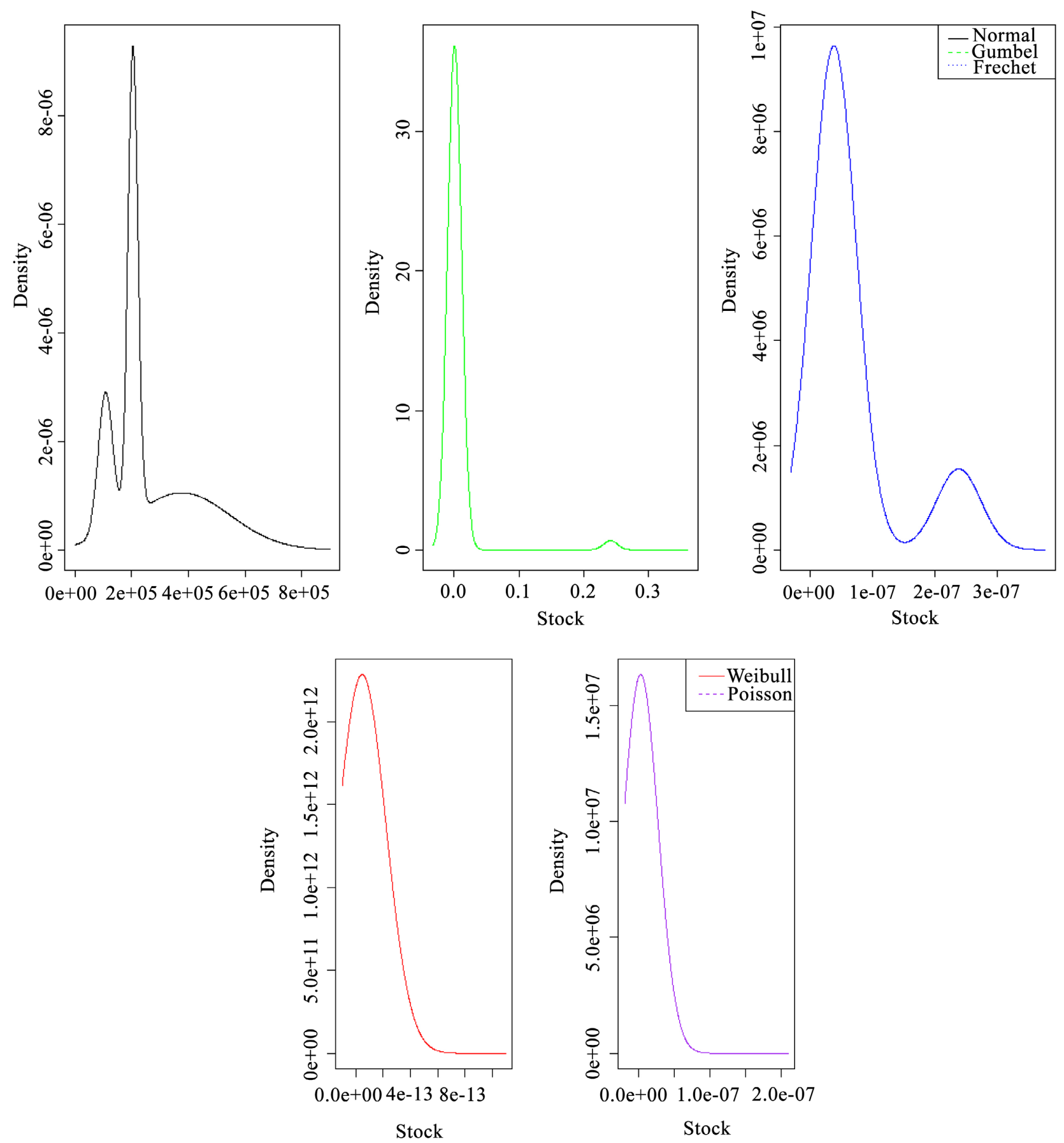

Figure 4. Distributional density plots of Poisson, Normal and EVDs for monthly number of stocks sold.

compare to all negative AICs and BICs of -8737.242 and $-8766.328 ;-8675.2303$ and $-8696.455 ;-3556.1$ and $-3547.4 ;-2099$ and -2092 for Gumbel-MAR $(2: 1$, 1), Fréchet-MAR (2:1, 1), Weibull-MAR (1) and Poisson-MAR (1) respectively. All together, the EVDs-MAR possessed smaller AICs and BICs compare to the linear models, other regime-switching non-linear models with Normal, Poisson, Gumbel, Fréchet and Weibull distributions. It was also noted that Gumbel-MAR $(2: 1,1)$ outstripped other linear models subjected to, as well as best fitted when 
Table 4. Fitted of EVDs-MAR and SETAR models to the monthly number of stocks.

\begin{tabular}{|c|c|c|c|c|c|c|c|c|}
\hline Stock & $\begin{array}{c}\% \text { in } \\
\text { low regime }\end{array}$ & $\begin{array}{l}\% \text { in high } \\
\text { regime }\end{array}$ & AIC & BIC & $\begin{array}{l}\text { Residuals } \\
\text { variance }\end{array}$ & MAPE & $\begin{array}{l}\text { Threshold } \\
\text { Value }\end{array}$ & \\
\hline $\begin{array}{c}\text { Norm-SETAR } \\
\quad(2: 2,1)\end{array}$ & $32.76 \%$ & $67.24 \%$ & 1432 & 1445 & 0.0031 & $52.59 \%$ & 191719 & \\
\hline $\begin{array}{c}\text { Gumbel-SETAR } \\
(2: 2,1)\end{array}$ & $16.31 \%$ & $83.69 \%$ & -7938 & -7955 & 0.0123 & $21.29 \%$ & 0.5028 & \\
\hline $\begin{array}{l}\text { Fréchet-SETAR } \\
\qquad(2: 2,2)\end{array}$ & $17.58 \%$ & $82.42 \%$ & -7102 & -7113 & 0.0007 & $25.47 \%$ & 0.6291 & \\
\hline $\begin{array}{c}\text { Weibull-SETAR } \\
\qquad(2: 2,2)\end{array}$ & $12.49 \%$ & $87.51 \%$ & -7810 & -7829 & 0.0093 & $23.36 \%$ & 0.8180 & \\
\hline $\begin{array}{c}\text { Poisson-SETAR } \\
(2: 2,2)\end{array}$ & $11.67 \%$ & $88.33 \%$ & -7078 & -7097 & 0.0064 & $24.67 \%$ & 0.4257 & \\
\hline MAR & $\begin{array}{l}\text { Mixing Weight } \\
\left(\omega_{k}\right) \text { per regime }\end{array}$ & AIC & BIC & $\mu_{k} / \alpha_{k}$ & $\beta_{k}$ & $\sigma_{k}$ & Log.Lik & $\begin{array}{c}\text { EM Test } \\
\text { for No. } \\
\text { regime }\end{array}$ \\
\hline $\begin{array}{c}\text { Norm-MAR } \\
(3: 2,1,3)\end{array}$ & $\begin{array}{c}(0.6680 \\
0.1883,0.1437)\end{array}$ & 1517.1827 & 1523.166 & $\begin{array}{c}(612.62, \\
253.85,-185.37)\end{array}$ & - & $\begin{array}{c}(262.47 \\
89.72,220.49)\end{array}$ & -733.2816 & $\begin{array}{c}3.3 \approx 3 \\
(0.0000)\end{array}$ \\
\hline $\begin{array}{c}\text { Gumbel-MAR } \\
(2: 1,1)\end{array}$ & $(0.4891,0.5109)$ & -8737.242 & -8766.328 & $(0.0046,0.0089)$ & $(0.0004,0.0042)$ & $\begin{array}{l}(0.02 \\
0.44)\end{array}$ & 5634.239 & $\begin{array}{l}2.0 \approx 2 \\
(0.0000)\end{array}$ \\
\hline $\begin{array}{c}\text { Fréchet-MAR } \\
(2: 1,1)\end{array}$ & $(0.4988,0.5012)$ & -8675.2303 & -8696.455 & $(0.0002,0.00246)$ & $(0.0004,0.0042)$ & $\begin{array}{l}(2.67, \\
4.89)\end{array}$ & 4397.435 & $\begin{array}{l}1.89 \approx 2 \\
(0.004)\end{array}$ \\
\hline Weibull-MAR (1) & $0.9999 \approx 1$ & -3556.1 & -3547.4 & 0.0009 & 0.0045 & 0.0001 & 1780.97 & - \\
\hline Poisson-MAR (1) & $0.9999 \approx 1$ & -2099 & -2092 & 0.00039 & - & 0.002 & 1051.98 & - \\
\hline
\end{tabular}

subjected as random noise in the category of MAR models for the discrete monthly stocks sold series. This possibly suggested that Gumbel might be a paragon random noise for time series for MAR model when fitting strictly discrete time-varying contaminated series. Below is the coefficient table for the AR regime-switching processes as well as the fitted model for the better Gumbel-MAR $(2: 1,1)$ (Table 5$)$.

$$
\begin{gathered}
\operatorname{Gumbel-MAR}(2: 1,1) \approx(0.4891,0.0046,0.0004) \Phi\left(\frac{y_{t}-0.0021 y_{t-1}}{0.02}\right) \\
+(0.5109,0.0089,0.0042) \Phi\left(\frac{y_{t}-0.0219 y_{t-1}}{0.44}\right) \\
\hat{Y}_{t}=\left\{\begin{array}{lll}
y_{t}+0.0021 y_{t-1} & \ni & \eta_{1}=0.4891 ; \mu_{1}=0.0046 ; b_{1}=0.0004 \\
y_{t}+0.0219 y_{t-1} & \ni & \eta_{2}=0.5109 ; \mu_{2}=0.0089 ; b_{2}=0.0042
\end{array}\right. \\
\sum_{k=1}^{2} \eta_{k}=0.4891+0.5109 \approx 1
\end{gathered}
$$

It is to be noted that all the AR coefficients in each regime are stationary, that is, $\phi_{k, i} \in(0,1)$. Though, it has been ascertained and proved by Boshnakov 


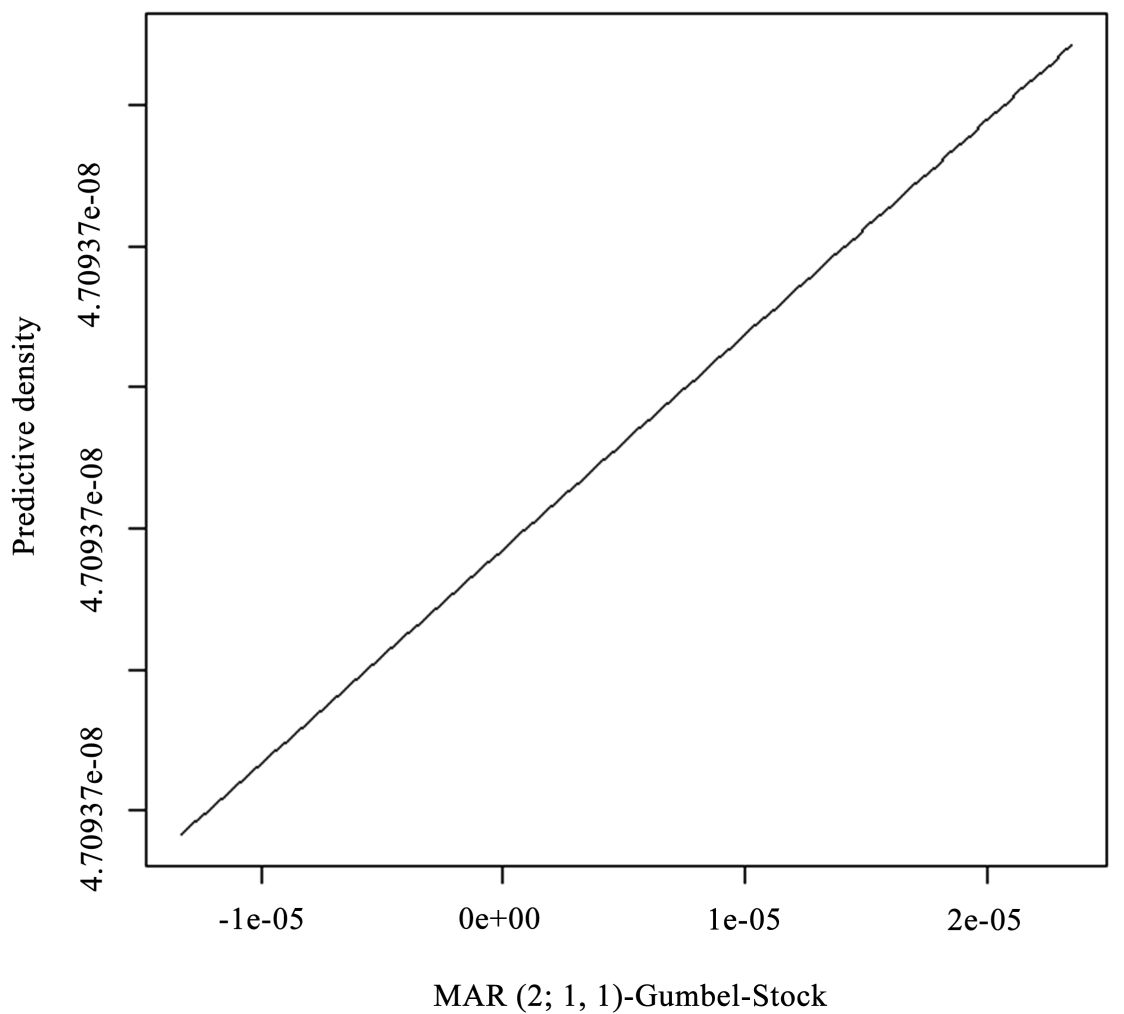

Figure 5. The predictive density curve for the Gumbel-MAR $(2: 1,1)$.

Table 5. Components of the AR Processes for the fitted Gumbel-MAR $(2: 1,1)$ discrete monthly number of stocks sold.

\begin{tabular}{cccc}
\hline Coefficients & $\eta_{k}$ & $\phi_{11}$ & $\phi_{21}$ \\
\hline \multirow{2}{*}{ Comp. 1 } & 0.4891 & 0.021 & - \\
& $(0.0021)$ & $(0.0330)$ & 0.0219 \\
Comp. 2 & 0.5109 & & $(0.0026)$ \\
\hline
\end{tabular}

(2006) that if non-stationary AR coefficient (s) in one or two regimes is/are mixed with stationary AR process(es), the whole mixture system becomes stationary.

Figure 5 is for the density predictive curve for the fitted Gumbel-MAR $(2: 1,1)$ for five years forecast of the monthly stocks sold.

\section{Conclusion}

In this research, four new novel statistical, time series and regime-shifting models were formulated to study the transitional shifting mechanisms of time-varying financial returns (monthly number of stocks). These four statistical and non-linear time series models were analyzed qualitatively and quantitatively. These four statistical models can contribute immensely to any contaminated series with outliers, extreme valued, excess kurtosis, excess skewness and non-normally distributed data in modelling of time constraint. In conclusion, Gumbel random 
noise provided the minimum error term for MAR model, with Gumbel-MAR $(2: 1,1)$ being the generalization for the non-linear tenure-switching. Though, the Fréchet-SETAR $(2: 2,2)$ for the two switching-tenure innovation coincides with MAR the generalization of Gumbel-MAR $(2: 1,1)$ model that also fitted two switching-tenures, the latter failed to withstand the generalization performance of the formal. The merit of the proposed nonlinear and time-switching time series models was not only peculiar to the enablement to establish the number of switching tenures associated to a certain series, but also to ascertain their switching autoregressive processes with their associated lag and coefficients.

\section{Acknowledgements}

Our self-effacement goes to the Central Bank of Nigeria as well as the Nigeria Stock Exchange for their unflinching assistance for providing the dataset used in this research.

\section{Conflicts of Interest}

The authors declare no conflicts of interest regarding the publication of this paper.

\section{References}

[1] Geetha, C., Mohidin, R., Chandran, V.V. and Chong, V. (2011) The Relationship between Inflation and Stock Market: Evidence from Malaysia, United States and China. International Journal of Economics and Management Sciences, 1, 1-16.

[2] Uwubanmwen, A. and Eghosa, I.L. (2015) Inflation Rate and Stock Returns: Evidence from Nigerian Stock Market. International Journal of Business and Social Science, 6, 155-167.

[3] Petrica, A.C., Stancu, S. and Tindeche, A. (2016) Limitation of ARIMA Models in Financial and Monetary Economics. Theoretical and Applied Economics, 23, 19-42.

[4] Qiu, M. and Song, Y. (2016) Predicting the Direction of Stock Market Index Movement Using an Optimized Artificial Neural Network Model. PLoS ONE, 11, Article ID: e0155133. https://doi.org/10.1371/journal.pone.0155133

[5] Nguyen, N. (2018) Hidden Markov Model for Stock Trading. International Journal Financial Studies, 6, Article No. 36. https://doi.org/10.3390/ijfs6020036

[6] Cao, C.Q. and Tsay, R.S. (1992) Nonlinear Time-Series Analysis of Stock Volatilities. Journal of Applied Econometrics, 7, S165-S185.

https://doi.org/10.1002/jae.3950070512

[7] Abrahamson, A. and Simon, C. (2018) Stock Market Anomalies: The Day-of-theWeek-Effect, an Empirical Study on the Swedish Stock Market: A GARCH Model Analysis. Doctoral Thesis, Jönköping University, International Business School, Sweden.

[8] Kalliovirta, L., Meitz, M. and Saikkonen, P. (2012) A Guassian Mixture auto Regresive Model for Univariate Series. Journal of Time Series Analysis, 36, 247-266. https://doi.org/10.1111/jtsa.12108

[9] Batta. N. (2014) Relationship between Trading Volume and Stock Return Volatility: Evidence from Nairobi Securities Exchange. Master's Thesis, University of Nairobi, Nairobi. 
[10] Giovanis, E. (2014) The Turn-of-the-Month-Effect: Evidence from Periodic Gen eralized Autoregressive Conditional Heteroskedasticity (PGARCH) Model. International Journal of Economic Sciences and Applied Research, 7, 43-61. https://doi.org/10.2139/ssrn.2479295

[11] McElroy, T. and Monsell, B. (2015) Model Estimation, Prediction, and Signal Extraction for Non-Stationary Stock and Flow Time Series Observed at Mixed Frequencies. Journal of the American Statistical Association, 110, 1284-1303. https://doi.org/10.1080/01621459.2014.978452

[12] Uzuke, C.A., Obiora-Ilouno, H.O., Eze, F.C. and Daniel, J. (2016) Time Series Analysis of All Shares Index of Nigerian Stock Exchange: A Box-Jenkins Approach. International Journal of Sciences, 5, 24-38.

[13] Wong, C.S. (1998) Statistical Inference for Some Nonlinear Time Series Models. Ph.D Thesis, University of Hong Kong, Hong Kong.

[14] Wong, C.S. and Li, W.K. (2000) On a Mixture Autoregressive Model. Journal of Royal Statistical Society, Series B, Statistical. Methodology, 62, 95-115. https://doi.org/10.1111/1467-9868.00222

[15] Bartlett, A. and McCormick, W. (2012) Estimation for Nonnegative First-Order Auto regressive Processes with an Unknown Location Parameter. Applied Mathematics, 3, 2133-2147. https://doi.org/10.4236/am.2012.312A294

[16] McCormick, W.P. and Mathew, G. (1993) Estimation for Non-Negative Autoregressive Processes with an Unknown Location Parameter. Journal of Time Series Analysis, 14, 71-92. https://doi.org/10.1111/j.1467-9892.1993.tb00130.x. 